\title{
Enhanced saccharification of lignocellulosic agricultural biomass and increased bioethanol titre using acclimated Clostridium thermocellum DSM1313
}

\author{
M. Nisha ${ }^{1} \cdot$ K. Saranyah ${ }^{1} \cdot$ Mukund Shankar $^{2} \cdot$ L. M. Saleena ${ }^{1}$
}

Received: 17 August 2016/Accepted: 7 January 2017/Published online: 13 April 2017

(c) The Author(s) 2017. This article is an open access publication

\begin{abstract}
Consolidated bioprocess assures an efficient lignocellulosic conversion to fermentable sugars and subsequently to bioethanol. Such a single-step hydrolysis and anaerobic fermentation was achieved with acclimated Clostridium thermocellum DSM 1313 on different mildly pre-treated agricultural lignocellulosic residues without any additional enzymes/and strains. Acclimation was achieved by serially sub-culturing in increasing concentration of individual substrates, such as rice husk, sugarcane bagasse, and banana pseudostem in the standard media, with cellobiose as an adjunct. The acclimated cellulolytic thermophile exhibited an early log phase entry with enhanced growth compared to the direct inoculation experiments with unacclimated culture. Around $672 \mathrm{mg} / \mathrm{g}$ of reducing sugar was produced from sugarcane bagasse media and $636 \mathrm{mg} / \mathrm{g}$ from rice husk media and $513 \mathrm{mg} / \mathrm{g}$ from banana pseudostem media with the acclimated organism. Bioethanol production also doubled in experiments with serially acclimated cultures, with a maximum
\end{abstract}

L. M. Saleena

saleena.m@ktr.srmuniv.ac.in

M. Nisha

nisha.m53@gmail.com

K. Saranyah

ksaranyah.biotech@gmail.com

Mukund Shankar

mukund.20@gmail.com

1 Department of Biotechnology, School of Bioengineering, SRM University, Kattankulathur, Chennai, Tamil Nadu 603203, India

2 Department of Chemical Engineering, School of Bioengineering, SRM University, Kattankulathur, Chennai, Tamil Nadu 603203, India of 1.21 and $1.0 \mathrm{~g} / \mathrm{L}$ ethanol titre from sugarcane bagasse and rice husk, respectively. The serial acclimation experiments have increased the saccharification potentials of the organism towards the respective lignocellulosic substrates and also enhanced the bioethanol production.

Keywords Clostridium thermocellum - Acclimation . Lignocellulose $\cdot$ Cellobiose $\cdot$ Reducing sugars $\cdot$ Ethanol
Abbreviations
LCS Lignocellulosic substrate
BP Banana pseudostem
RH Rice husk
SB Sugarcane bagasse
$f$.RS Fraction of reducing sugar

\section{Introduction}

The first-generation biofuels have promoted edible crops as feedstocks for bioethanol production. The seasonal, regional, and rate of growth variations of the edible feed stocks have been reported to lead inconsistent supply for bioethanol production (Kim and Dale 2004). Today, secondgeneration biofuels are considered as advanced biofuels, since the feedstock generally used is not food crops, such as in first-generation biofuels. Various methods, such as physico-chemical/and enzymatic hydrolytic techniques, have been used for converting lignocellulosic composite to reducing sugars for bioethanol production. The widely adopted pre-treatments for lignocellulosic substrates (LCSs), such as steam explosion, acid hydrolysis, and ammonia fibre expansion, are often expensive and limit 
commercialization. In addition, in most cases, pre-treatments generate toxic substances necessitating a detoxification stage before hydrolysis (Maki et al. 2009; Brodeur et al. 2011).

Microbial hydrolysis is a promising approach that reduces time and cost in LCS hydrolysis in comparison with the non-microbial processing techniques. Only a few known microorganisms have the capability to degrade and hydrolyse recalcitrant plant biomass. Cellulolytic thermophiles are increasingly researched due to the high tolerance level to fluctuating $\mathrm{pH}$, temperature, and environmental changes. Their enzymes take in a crucial role in industries due to its thermo-stability (Pandey 2011). Clostridium thermocellum is an anaerobic, spore-forming cellulolytic thermophile producing multi-enzyme complex consisting of various hydrolytic enzymes as well as extracellular individual free enzymes. Having both these enzyme systems is a rare characteristic in the microbial world due to which $C$. thermocellum is an efficient biomass degrader along with ethanolgenic capabilities (Wilson 2011; Paye et al. 2016). Application of C. thermocellum simplifies the LCS bioprocess by simultaneous hydrolysis and fermentation of the hydrolysate within the same pot. The consolidated process involves least investment in enzyme production, cellulose hydrolysis, and enzyme recovery. However, irrespective of the technological advancements, the technical and economic constrains of converting the cellulosic material into fermentable sugars for the production of biofuels remain unsolved.

Researches are being carried out to enhance the hydrolytic capability of the wild strain $C$. thermocellum. $C$. thermocellum with superior hydrolytic capabilities is, however, reported to produce low-level cellulosomes (You et al. 2012). Even though the thermophile is claimed to completely convert pure cellulose to ethanol, it does not produce similar results with other cellulosic substrates (Pandey 2011). This work deduces the effect of microbial hydrolysis of $C$. thermocellum after acclimation towards LCSs. The efficiency of microbial hydrolysis was established in terms of released reducing sugars yield, recovery of residual LCS, and final ethanol concentration. Several mildly pre-treated agricultural residues were microbially hydrolysed using wild and acclimated $C$. thermocellum DSM1313 without any additional enzyme or chemical supplements.

\section{Materials and methods}

\section{Lignocellulosic substrates: collection and processing}

Rice husks (Oryza sativa L.), banana pseudostem (Musa paradisiaca L.), were collected after harvest from
Chunambedu, Kanchipuram district, Tamil Nadu, India. Sugarcane bagasse (Saccharum officinarum L.) was collected from Pavunjur, Kanchipuram district, Tamil Nadu, India. Collected samples were air-dried, chopped into fine pieces with kitchen aid mixer, and sifted through BS-410, mesh 36. Ground LCSs were then stored in zipper lock polypropylene plastic bags at room temperature until analysis and treatment.

\section{LCSs: preparation and pre-treatment}

Rice husk (RH), sugarcane bagasse (SB), and banana pseudostem (BP) were washed with water and dried overnight at $50{ }^{\circ} \mathrm{C}$. Dried samples were pre-treated with $250 \mathrm{mM} \mathrm{NaOH}$ solution (Kiyoshi et al. 2015) for $20 \mathrm{~min}$ at $121^{\circ} \mathrm{C}$. Treated LCSs were neutralised with repeated distilled water wash and dried in $50{ }^{\circ} \mathrm{C}$ (overnight) and cooled in desiccator until constant weight was achieved.

\section{Field emission scanning electron microscope analysis}

Field emission scanning electron microscope (FESEM) samples were non-conductive, and the mode was low and extended low vacuum. The samples were spread on the carbon tape and analysed with an accelerating voltage of $20 \mathrm{kV}$ using FEI's Quanta 200 FEG.

\section{Fourier transform infrared spectroscopy analysis}

Samples were ground along with potassium bromide in the ratio $1: 10$ and pelleted under pressure to form uniformly spread thin discs $\sim 10 \mathrm{~mm}$ diameter and analysed by Fourier transform infrared spectroscopy (FTIR) (Cary 660; Agilent Technologies). Spectra were obtained by averaging 16 scans from 4000 to $400 \mathrm{~cm}^{-1}$ at $4 \mathrm{~cm}^{-1}$ resolution. The blank scan was an average of 64 .

\section{Colorimetric determination of cellulose}

The sample (500 mg) was homogenized with distilled water to which $5 \mathrm{~mL}$ acetic acid-nitric acid reagent was added and placed in a boiling water bath for $30 \mathrm{~min}$. Cooled sample was centrifuged ( $4250 \mathrm{~g}$ for $5 \mathrm{~min}$ ), and the pellets were washed with distilled water followed by the slow addition of $10 \mathrm{~mL}$ of $67 \% \mathrm{H}_{2} \mathrm{SO}_{4}(\mathrm{v} / \mathrm{v})$ and mixed intermittently. This sample was kept aside for $1 \mathrm{~h}$ and diluted to $100 \mathrm{~mL}$ and centrifuged $(1500 \mathrm{~g}$ for $10 \mathrm{~min}$ ) if any precipitate or turbidity present. For the assay, $0.1 \mathrm{~mL}$ of diluted sample was taken and $4.9 \mathrm{~mL}$ distilled water was added. $10 \mathrm{~mL}$ ice cold anthrone reagent $(0.2 \mathrm{~g}$ in $100 \mathrm{~mL}$ conc. $\mathrm{H}_{2} \mathrm{SO}_{4}$ ) was added keeping the tube in an ice bath. It was then kept in boiling water bath for $15 \mathrm{~min}$, cooled 
rapidly in ice bath. The absorbance of the blue green colour developed was read at $630 \mathrm{~nm}$ against a reagent blank (Updegraff 1969).

\section{Culture and storage of strain}

Clostridium thermocellum DSM1313 was purchased from Leibniz Institute DSMZ-German Collection of Microorganisms and Cell Cultures. Stock cultures were maintained in DSM 122 media with $5 \mathrm{~g} / \mathrm{L}$ of cellobiose as the carbon source. Long-term culture storage was done by re-suspending the pelleted cultures in DSM122 cellulose $(10 \mathrm{~g} /$ L) media and $50 \% \mathrm{v} / \mathrm{v}$ glycerol at $-80{ }^{\circ} \mathrm{C}$.

\section{Inoculum preparation}

All aseptic transfers pertaining to anaerobic cultivation were performed in an anaerobic hood flushed with nitrogen gas. $10 \%$ (v/v) of DSM122 cellulose medium (passaged for three batches) was the initial seed considered in all experiments unless otherwise mentioned.

\section{Microbial hydrolysis and fermentation of agricultural substrates using Clostridium thermocellum}

\section{Experimental setup I: direct inoculation}

Cellulose ingredient in DSM 122 media was substituted by LCS under study. They were weighed and added based on their respective cellulose content gram (g) equivalent to recommended DSM cellulose concentration. $100 \mathrm{~mL}$ vials having $70 \mathrm{~mL}$ of the media were inoculated with $7 \mathrm{~mL} C$. thermocellum seed inoculum. All vials were flushed with nitrogen gas and sealed with butyl rubber stoppers and aluminium crimps. Concurrently, all the vials were incubated for $120 \mathrm{~h}$ at $60{ }^{\circ} \mathrm{C}$ with an initial $\mathrm{pH}$ of 7.4. Reducing sugars and growth were recorded every $24 \mathrm{~h}$. DSM 122 cellulose medium was the control. Triplicates were kept for all batches (Fig. 1).

\section{Experimental setup II: serial acclimation experiment}

Four different serial acclimation experiment (SAE) series viz, SAE1, SAE2, SAE3, and SAE4 were formulated based on the varying $(\mathrm{g})$ equivalent quantity of LCS cellulose. Cellobiose was added as an adjunct to suffice the $10 \mathrm{~g} / \mathrm{L}$ of total carbohydrate content as recommended in DSM 122 media composition. The whole experimental setup was carried out by serially sub-culturing from SAE1 to SAE4.

Formulation of the series was:
1. SAE1: $7.5 \mathrm{~g} / \mathrm{L}$ of cellobiose and LCS ( $\mathrm{g}$ ) equivalent to $2.5 \mathrm{~g} / \mathrm{L}$ of pure cellulose;

2. SAE2: $5.0 \mathrm{~g} / \mathrm{L}$ of cellobiose and LCS ( $\mathrm{g}$ ) equivalent to $5.0 \mathrm{~g} / \mathrm{L}$ of pure cellulose;

3. SAE3: $2.5 \mathrm{~g} / \mathrm{L}$ of cellobiose and LCS (g) equivalent to $7.5 \mathrm{~g} / \mathrm{L}$ of pure cellulose;

4. SAE4: LCS (g) equivalent to $10.0 \mathrm{~g} / \mathrm{L}$ of pure cellulose.

Here, uniform volume of $70 \mathrm{~mL}$ in $100 \mathrm{~mL}$ serum vials was maintained for all trials. Inoculum for SAE1 and experimental setup1 was similar. Strategy involved a serial passaging of $96 \mathrm{~h}$ old SAE1 to SAE2 medium with $10 \%$ (v/ v) inoculum. This inoculated medium after $96 \mathrm{~h}$ of incubation then served as a seed $(10 \% \mathrm{v} / \mathrm{v})$ for the next higher LCS concentration containing medium and so forth (SAE1 » SAE4). For every setup, the growth and reducing sugar concentrations were intermittently checked for every $24 \mathrm{~h}$. Triplicates were kept for all batches.

\section{Growth analysis}

Grown cultures were vigorously mixed on a vortex mixer (at room temperature) to free the adhered cells from LCS, and the particles were allowed to settle for an hour (Fleming and Quinn 1971). Growth spectrometrically measured at $600 \mathrm{~nm}$.

\section{Colorimetric determination of reducing sugar}

Reducing sugar analysis of all samples was conducted by 3,5-dinitrosalicylic acid assay (Miller 1959). $171 \mu \mathrm{L}$ of DNS reagent was added to $9 \mu \mathrm{L}$ sample supernatant (centrifuged at $9000 \mathrm{~g}, 10 \mathrm{~min}$ ) in a 96 well non-skirted PCR plate. The reaction mix was heated to $95{ }^{\circ} \mathrm{C}$ for $5 \mathrm{~min}$ in a thermocycler and subsequently held at $20^{\circ} \mathrm{C}$ for $10 \mathrm{~min}$ for cooling and read at $540 \mathrm{~nm}$. Total reducing sugar concentration was calculated from linear regression of the pre-assayed standard glucose and expressed as $\mathrm{g} / \mathrm{L}$.

\section{Data analysis and interpretation of the experimental setup I and II}

Fraction values (f.RS)

To evaluate the hydrolysate based on release of reducing sugar, fraction values ( $f$.RS) of the total reducing sugars (g/ L) of every $24 \mathrm{~h}$ from a 5 day batch was calculated:

$f . \mathrm{RS}=\frac{\text { total reducing sugar of } \mathrm{nth} \text { hour }}{\text { total reducing sugar at } 0 \text { th hour }}$.

Total reducing sugars in the broth when inferred as a fraction indicates consumption or release of sugars into the 
Fig. 1 FESEM micrographs. a Untreated SB, a1 treated SB, detached fibrils are depicted in the circle. b Untreated BP, b1 treated BP, lignin particles attached to the fibres depicted in the circle. c Untreated RH, c1 treated RH. Magnification scale bar is illustrated in each image
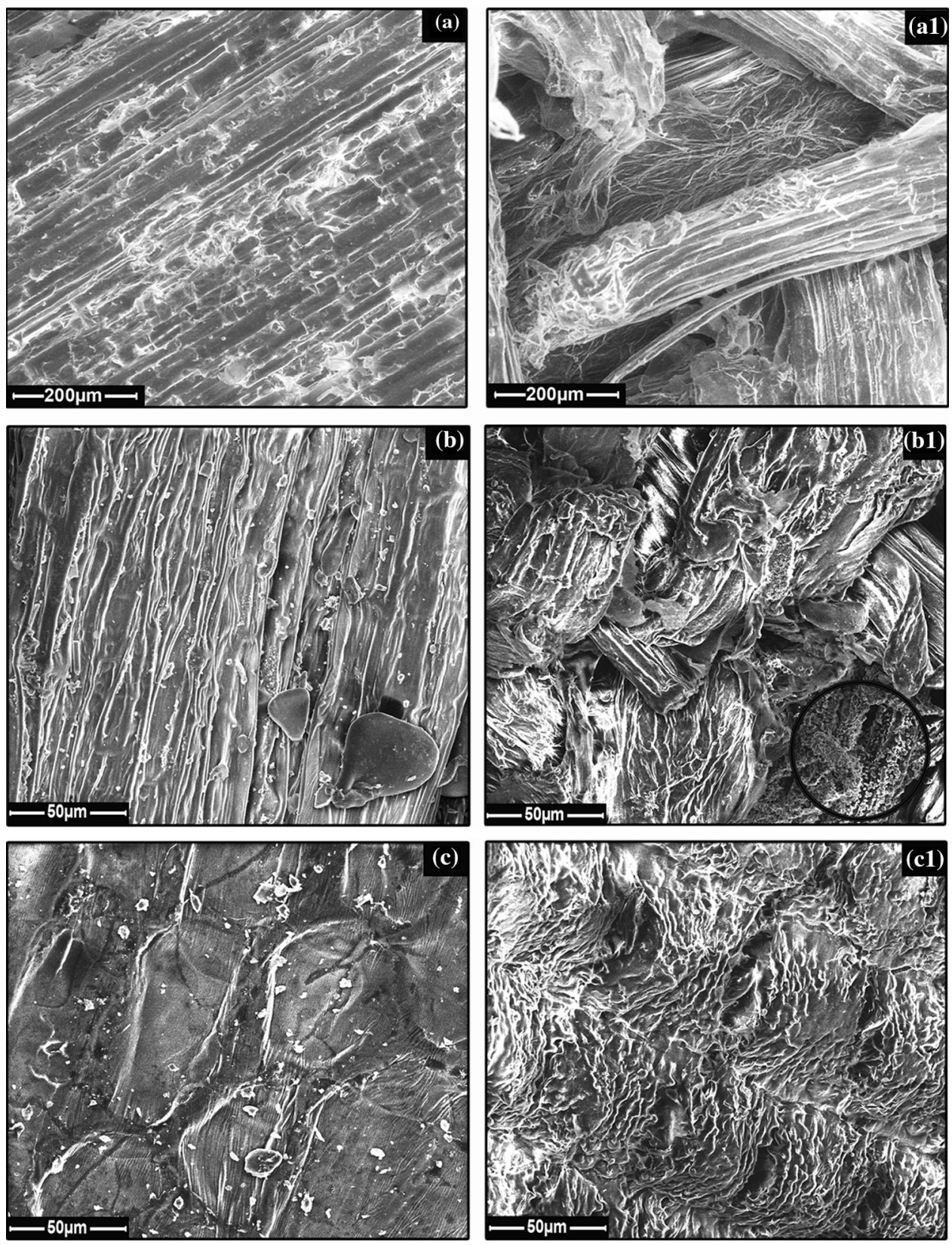

media at a particular time of analysis. $f$. RS values $>1.0$ signify release of reducing sugars, and $f$. RS values $<1.0$ signify consumption of the available, reducing sugars at that particular period. The rate of consumption will be equal to the rate of release when $f . \mathrm{RS}=1$ under a normal cell growth pattern (Fig. 3b).

\section{Determination of residual LCS}

The remnant LCS feed in the broth after fermentation was pelleted at $5000 \mathrm{~g}$ for $15 \mathrm{~min}$. The pellets were washed with double distilled water twice and filtered. The residuals were dried and cooled in a desiccator until a constant weight was achieved for gravimetric analysis (Dharmagadda et al. 2010).

\section{Chromatographic analysis}

Identification of the mono-sugars from the derived hydrolysate

HPLC (UFLC LC-20 AD; Shimadzu RID-10A detector) analyses were performed to identify mono-sugars released at the end of 5 day microbial hydrolysis. Luna NH2 100A column having a length of $150 \mathrm{~mm}$, ID of $4.6 \mathrm{~mm}$, and pore size of $5 \mu \mathrm{m}$ was considered. $80 \%$ acetonitrile in 
water was used as mobile phase with a flow rate adjustment to $3 \mathrm{~mL} / \mathrm{min}$ and temperature at $30{ }^{\circ} \mathrm{C}$ (Lopez Hernandez et al. 1998). $10 \mu \mathrm{L}$ of sample was injected. Glucose, galactose, arabinose, mannose, xylose, fructose (Supelco Monosaccharides kit), and cellobiose were the major standards used for the study.

\section{Ethanol concentration}

Ethanol concentration was analysed using reversed phase HPLC. Analyses were performed in HPLC 1260 infinity (Agilent Technologies) with equipped with G1329B autosampler. Water was used as a mobile phase in a $4.6 \times 250 \mathrm{~mm}$ Zorbax Eclipse Plus C18 column with $5 \mu \mathrm{m}$ pore size. $4 \%$ acetone was added to the mobile phase as a UV absorbing agent. Ethanol was detected as a negative peak. The intensity was measured with a PDA-UV detector at $235 \mathrm{~nm}$. Samples were prepared by centrifuging the broth; $50 \mathrm{~g}$ of supernatant was distilled $\left(79 \pm 1{ }^{\circ} \mathrm{C}\right)$ to collect a uniform weight of $10 \mathrm{~g}$. $10 \mu \mathrm{L}$ of which was subjected to HPLC. The values were calculated from linear regression of the standards (Nisha et al. 2016).

\section{Results and discussion}

This study explains the strategy of developing hydrolysate rich in mono-sugars from LCS for consequent ethanol production using Clostridium thermocellum. Pre-treatments, such as a steam explosion, acid/enzyme, or in combinations, are reported for lignin removal as well as for saccharification of LCS. Since $C$. thermocellum has feruloyl esterases which can release lignin from hemicellulose (Blum et al. 2000) and also due to the presence of a welldefined cellulolytic complex comprising of 72 different proteins along with 25 free enzymes (Taylor et al. 2009), only mild pre-treatments with dilute $\mathrm{NaOH}$ were considered to process the LCSs just enough to expose the cellulosic moieties.

\section{Pre-treatment of LCS}

To develop robust microbes for efficient biomass conversion and bioethanol production, the quality of the pretreated biomass is of prior importance. Naturally occurring biomass has a well-structured tightly arranged heterogeneous biochemical matrix with small-sized pores making it inaccessible to hydrolytic enzymes to act upon glycosidic bonds. This necessitates a pre-treatment before to hydrolysis to increase the porosity, hemicellulose solubilisation, lignin solubilisation, and lignin redistribution (Chaturvedi and Verma 2013). A physical process, such as grinding, before alkali pre-treatment is a necessity for increasing the surface area for microbial adherence and to decrease the cellulose crystallinity (Taherzadeh and Karimi 2008). Such physical parameters are a major contributor in improvising the conversion of biomass residues to fermentable sugars. Here, the finely ground substrates were sieved to ensure uniform granularity of maximum size of $\sim 400 \mu \mathrm{m}$. Under study, the highest cellulose content was estimated in BP $(53.7 \pm 1.7 \%)$ followed by $\mathrm{SB}(42.3 \pm 1.4 \%)$ and $\mathrm{RH}$ $(26.2 \pm 1.34 \%)$. LCSs were pre-treated with $250 \mathrm{mM}$ of $\mathrm{NaOH}$ for the selective disruption of lignin with less carbohydrate degradation and minimal inhibitor formations (Kumar et al. 2009). Wang et al. (2010) reported that $1 \%$ $\mathrm{NaOH}$ pre-treatment for $30 \mathrm{~min}$ at $121{ }^{\circ} \mathrm{C}$ was sufficient to achieve a maximum lignin removal and gave significantly higher total reducing sugars than that with $3 \% \mathrm{NaOH}$ (Wang et al. 2010).

\section{FESEM and FTIR analysis of the pre-treated substrates}

The uniformity and the highly ordered patterns throughout the fibre length of all the substrates were lost. The inner fibres of the treated SB were exposed to the outer surface which possibly enhanced the accessible area for microbial adherence. The uniformly arranged fibres aligned in parallel to the axis bundle were disturbed in treated BP samples. Detached lignin particles were apparently visible in the distorted BP samples. Untreated RH has a highly ordered arrangement of fibrils giving a very smooth appearance, whereas treated $\mathrm{RH}$ had a complete distortion sufficient enough to expose the inner regions (Fig. 1).

To identify the changes occurred in functional groups of the lignin cellulose moieties of the pre-treated biomass were analysed in FTIR. All the major peak ranges considered for this study are mentioned in Table 1. Less absorbance at peak 'a' of treated SB infers the disruption of $\mathrm{H}$ bond of cellulose hydroxyl group (Sindhu et al. 2010). Similar effects were also seen in fingerprints specific to lignin linkages (Table 2; Fig. 2a). In treated BP, peaks showed an overall drop in intensities. Noticeable dip in peak ' $\mathrm{g}$ ' is due to the bond disruption in glycoside linkage and aromatic groups of lignin (Guimarães et al. 2009). However, compared to other substrates, the decrease in bond intensity was not substantial (Table 2; Fig. 2b). The authors have not come across reports on delignification studies of $\mathrm{RH}$ with various alkaline pretreatments. In treated RH, the peaks of ' $\mathrm{f}$ ' were not detected and ' $b$ ', ' $g$ ', and ' $a$ ' showed reduced absorbance. One of the primary functional group related to lignin at peak ' $\mathrm{f}$ ' linking the hemicellulose has disappeared (Ndazi et al. 2007; Luduena et al. 2011) (Table 2; Fig. 2c). Among the three substrates, better performance was observed in SB and RH. 
Table 1 Assignment of major functional groups unique for lignin and cellulose in lignocellulosic substrates

\begin{tabular}{lllr}
\hline Absorption band location $\left(\mathrm{cm}^{-1}\right)$ & Assignment & Peak labels & References \\
\hline $3600-3100$ & H bond of OH group of cellulose & a & Pereira et al. (2014) \\
$2937-2918$ & - CH stretch of methyl and methylene groups of cellulose & b & Pereira et al. (2014) \\
$1731-1720$ & C=O stretch of ketone/aldehyde & c & Bilba et al. (2007) \\
$1428-1425$ & Aromatic ring vibrations of lignin & e & Bilba et al. (2007) \\
$1375-1320$ & Phenol hydroxyl stretch; lignin & g & Bilba et al. (2007) \\
$1167-1151$ & Ester bond stretch of lignin and carbohydrate & Bilba et al. (2007) \\
\hline
\end{tabular}

Table 2 FTIR spectroscopic results of untreated and treated SB, BP, and RH

\begin{tabular}{|c|c|c|c|c|c|c|c|c|c|c|c|c|c|}
\hline \multirow[t]{3}{*}{ Peak label } & \multicolumn{4}{|l|}{ SB } & \multicolumn{4}{|l|}{ BP } & \multicolumn{5}{|l|}{$\mathrm{RH}$} \\
\hline & \multicolumn{2}{|c|}{ Untreated } & \multicolumn{2}{|l|}{ Treated } & \multicolumn{2}{|c|}{ Untreated } & \multicolumn{2}{|l|}{ Treated } & \multicolumn{2}{|c|}{ Untreated } & \multicolumn{3}{|l|}{ Treated } \\
\hline & WN & $\mathrm{H}$ & WN & $\mathrm{H}$ & WN & $\mathrm{H}$ & $\mathrm{WN}$ & $\mathrm{H}$ & WN & $\mathrm{H}$ & WN & $\mathrm{H}$ & \\
\hline $\mathrm{a}$ & 3393.3 & 1.505 & 3403.0 & 0.690 & 3409.8 & 1.864 & 3407.8 & 1.022 & 3415.2 & 2.605 & 3406.4 & & 1.316 \\
\hline $\mathrm{b}$ & 2931.1 & 0.645 & 2930.3 & -0.061 & 2918.8 & 1.207 & 2924.8 & 0.525 & 2920.4 & 1.844 & 2919.7 & & 0.963 \\
\hline $\mathrm{c}$ & 1731.0 & 0.488 & $\mathrm{x}$ & & $\mathrm{x}$ & & $\mathrm{x}$ & & 1514.7 & 1.501 & $\mathrm{x}$ & & \\
\hline $\mathrm{e}$ & 1426.2 & 0.684 & 1427.9 & 0.043 & 1426.7 & 1.737 & $\mathrm{x}$ & & $\mathrm{x}$ & & $\mathrm{x}$ & & \\
\hline$f$ & 1375.6 & 0.687 & 1319.4 & -0.162 & $\mathrm{x}$ & & $\mathrm{x}$ & & 1372.5 & 1.712 & $\mathrm{x}$ & & \\
\hline & 1324.9 & 0.664 & $\mathrm{x}$ & & 1324.9 & 1.330 & 1325.8 & 0.602 & $\mathrm{x}$ & & $\mathrm{x}$ & & \\
\hline g & 1159.4 & 1.188 & 1161.0 & 0.069 & 1158.2 & 1.517 & 1153.3 & 0.767 & 1162.1 & 2.624 & 1157.8 & & 1.183 \\
\hline
\end{tabular}

$W N$ peak at $\left(\mathrm{cm}^{-1}\right), H$ peak height (a.u.), $x$ peak not detected

\section{Clostridium thermocellum and its behavioural growth trends in DSM122 cellulose media and DSM122 cellobiose media}

The growth of $C$. thermocellum in the standard cellulose and cellobiose media was observed to be similar (Fig. 3a) with a gradual $\mathrm{pH}$ shift to acidic range. The final $\mathrm{pH}$ values were 5.3 and 5.7 in cellobiose and cellulose media, respectively. The increased growth in these standard media infers the substrate familiarity and also its selective nature of action on the respective substrates. Even though the same growing seed culture was inoculated into the aforementioned standard substrates, $1.6 \times$ reduction in the total reducing sugar content was observed in cellobiose media, whereas an increase of $1.25 \times$ from the initial reducing sugar content was observed in the cellulose media. The 120th hour $f$.RS values were found to be 0.541 indicating consumption of reducing sugars and a fraction of 1.25 release of reducing sugars in the respective standard media (Fig. 3b). 64\% of cellulose conversion was observed in cellulose media. Final ethanol concentration estimated was 0.54 and $1.14 \mathrm{~g} / \mathrm{L}$ for cellobiose and cellulose media, respectively, which were in concurrent to published data (Weimer and Zeikus 1977; Tripathi et al. 2010).

\section{Microbial hydrolysis and anaerobic fermentation}

\section{Experimental setup I: direct Inoculation}

In DSM122 cellulose media, the lag phase extended up to $36 \mathrm{~h}$ and attained a maximum $\mathrm{OD}_{600}$ within $120 \mathrm{~h}$ batch. However, for RH, SB, and BP, the growth of Clostridium thermocellum had lag phase extending up to 72,76 , and $84 \mathrm{~h}$, respectively. Slow growth depicts the reduced competency of Clostridium thermocellum in adapting to new lignocellulose substrate, although the seed culture was vibrantly growing in cellulose. Similar observation was described elsewhere when active cultures grown in avicel were inoculated into pre-treated wood (Lynd 1989). The $\mathrm{pH}$ shift across $120 \mathrm{~h}$ had an acidic trend similar to the DSM122 cellulose media. According to Hörmeyer et al. (1988), the degradation of pre-treated LCSs was found to be equally effective in non-pH-controlled shake flask, in in vitro experiments with cell-free culture supernatant and also in vivo (cellulolyses) with $\mathrm{pH}$ regulation in a laboratory fermenter (Hörmeyer et al. 1988).

Soluble sugars keep accumulating in the media cultured with any cellulolytic bacteria even after the growth ceases because of the continuous activity of extracellular cellulase enzymes (Desvaux et al. 2000). It was observed that after 
Fig. 2 a FTIR spectrum of untreated and treated SB. b FTIR spectrum of untreated and treated BP. c FTIR spectrum of untreated and treated $\mathrm{RH}$ a

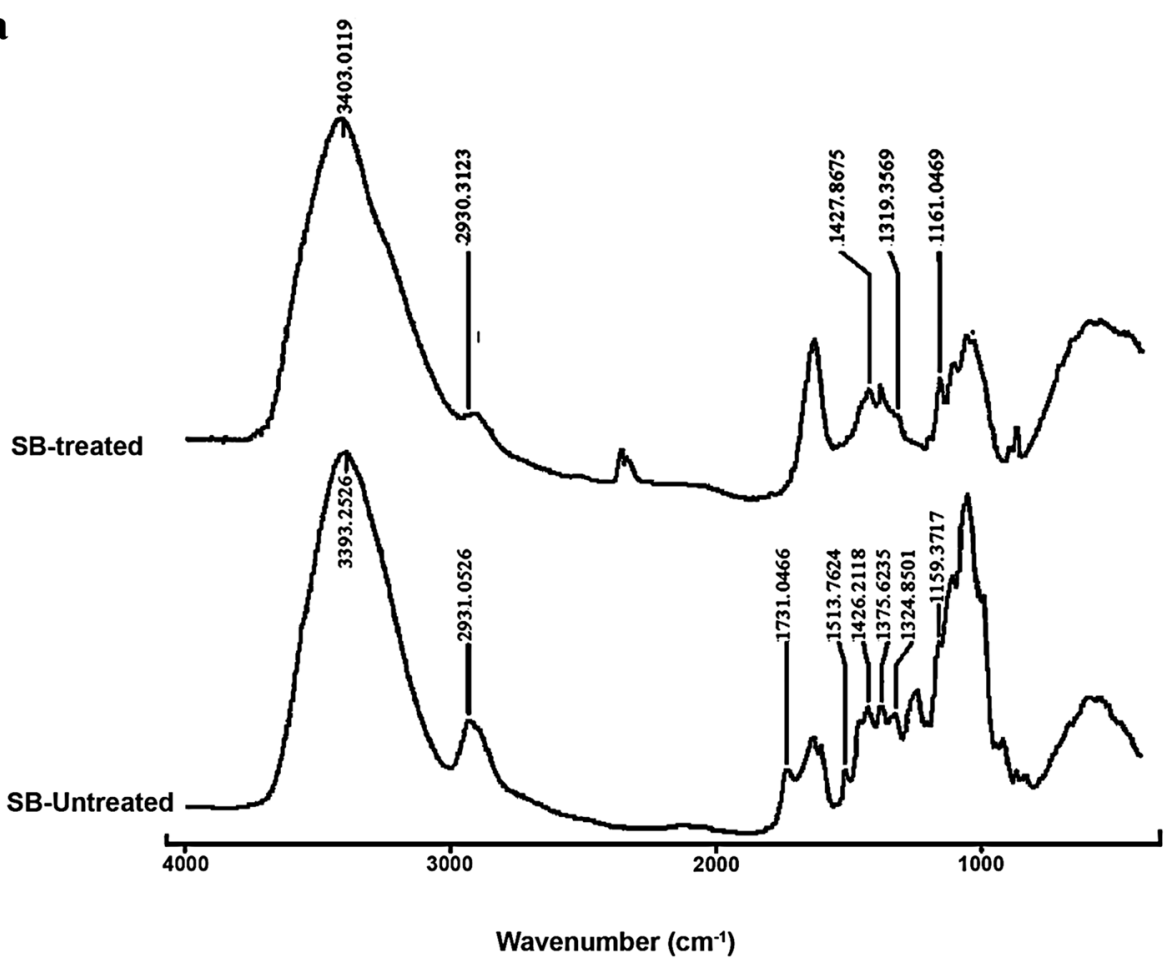

b

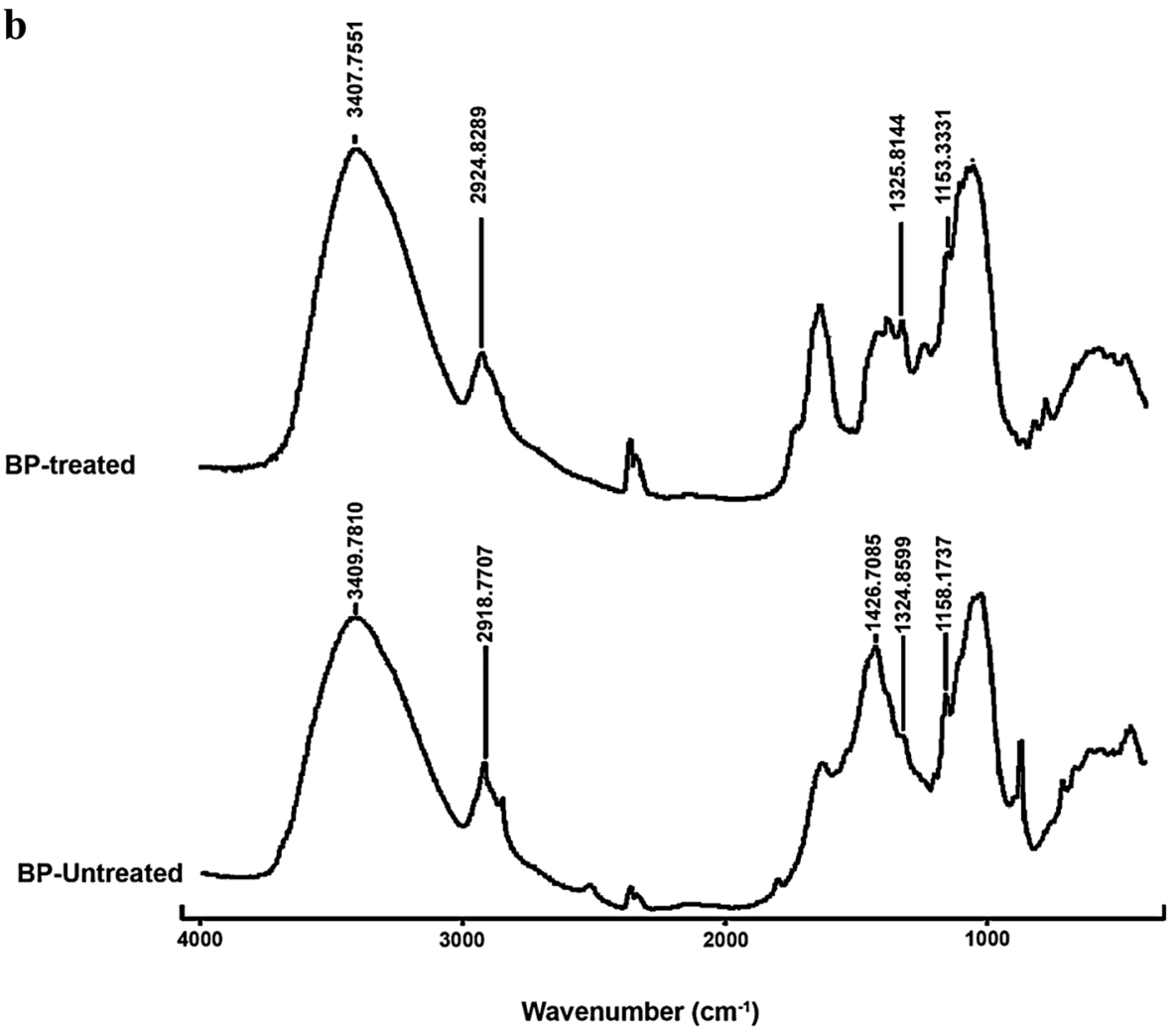

$120 \mathrm{~h}$, reducing sugar concentration in DSM122 cellulose medium increased by $25 \%$. However, sugar concentration in SB, BP, and RH medium decreased by $12.6,34.75$, and $27.17 \%$, respectively. $f$. RS value of the SB medium was observed to be close to unity (0.91), indicating that uptake rates of reducing sugar were similar to the released concentration. Whereas $f$.RS values of $\mathrm{RH}$ and BP medium were low, indicating sustenance of the organism in the 
Fig. 2 continued

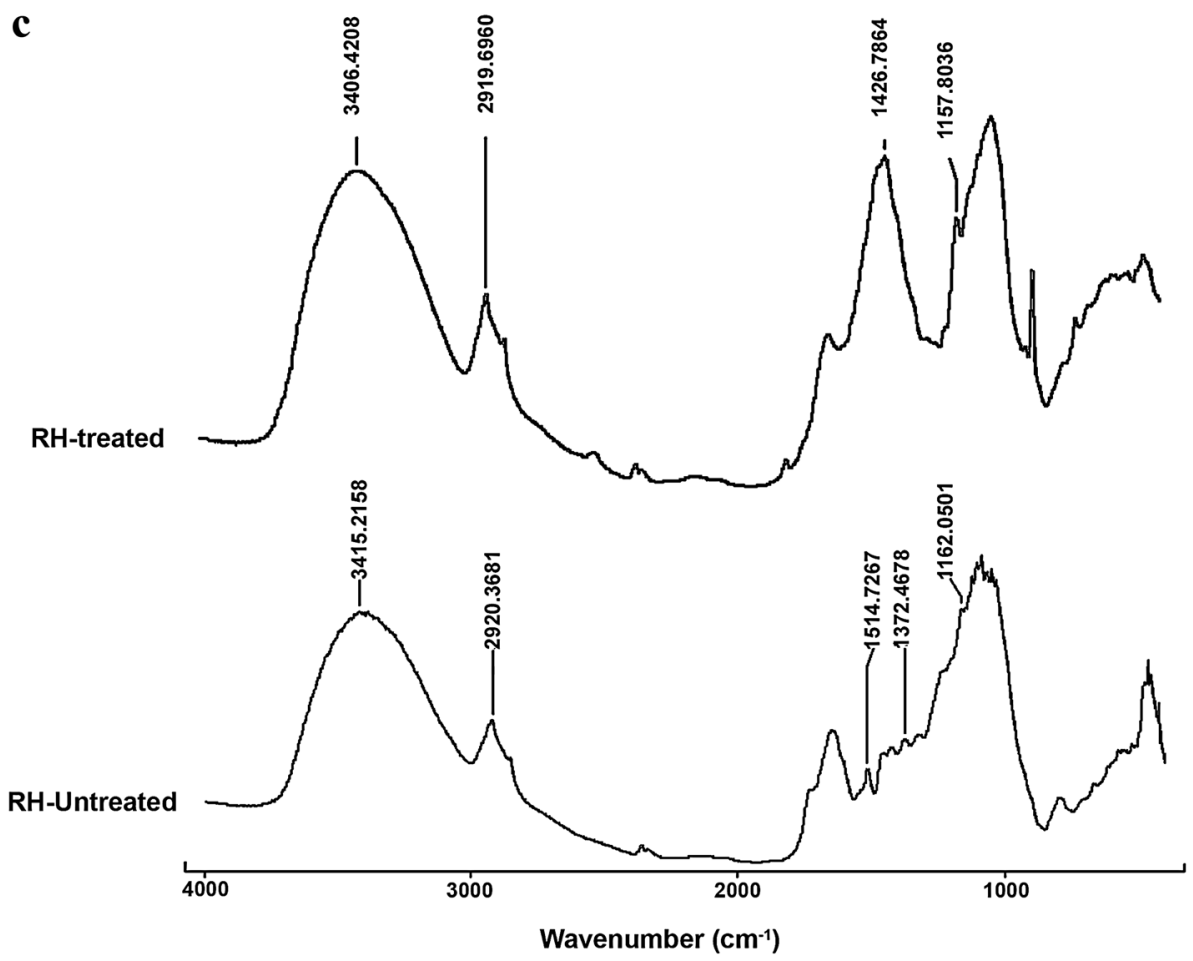

available sugars. Extending the incubation period (up to $168 \mathrm{~h}$ ) and/or sub-culturing further for several series did not increase the growth nor sugar concentration (data not shown) in any of the LCS media. Low growth of organism in all media resulted in low substrate conversion eventually with lower reducing sugar yield. Recovered residual biomass of different LCS media and its ethanol concentration is represented in Table 3.

\section{Experimental setup II: serial acclimation experiment}

It was observed that an actively growing culture struggled to grow when inoculated into LCS media (experimental setup I studies). Hence, in experimental setup II, residence interaction time of the microbe to the substrate was increased from 96 to $288 \mathrm{~h}$, by gradually sub-culturing it from least concentration of LCS with 2.5 (grams equivalent to cellulose) to $7.5 \mathrm{~g} / \mathrm{L}$ (gram equivalent to cellulose) with cellobiose as an adjunct. SAE1, SAE2, and SAE3 trials exhibited an early initiation of growth irrespective of the type of substrate, and these batches had maximum $\mathrm{OD}_{600}>0.9$. This rapid growth was due to the presence cellobiose, since it is a preferred assimilating disaccharide and a growth inducer for $C$. thermocellum than glucose (Das et al. 2013; Dionisi et al. 2014).

Even though the SAE1, SAE2, and SAE3 batches showed better growth, there was a dip in reducing sugar concentration from 0th to 120th hours across all substrates
(Table 4). This could be attributed to the organism utilising the available cellobiose for growth rather than the subjected LCSs. As the residence time increased across batches, an increment in the $f$.RS values was observed. Despite the absence the cellobiose adjunct in the SAE4 batches, SAE4-SB medium had $20.4 \%$ increase in reducing sugar concentration with $672 \pm 10 \mathrm{mg} / \mathrm{g}$ of cellulose equivalent released from the pre-treated substrate. $\mathrm{RH}$ was observed to have an $18.7 \%$ increase in reducing sugars with $636 \pm 15 \mathrm{mg} / \mathrm{g}$ of cellulose equivalent being released. The corresponding $f$.RS were $>1$ indicating the release of sugar upon hydrolysis. Contradictorily in BP medium, $f$.RS was $\sim 1.0$ with reducing sugar of $5.13 \pm 14 \mathrm{mg} / \mathrm{g}$ of cellulose equivalent. From the FTIR results, it was evident that less functional groups of LCS were disrupted, which in turn directly affected the release of reducing sugars with only $1.1 \%$ of increment from the 0th hour. Hence, the authors are of the opinion that mild alkali pre-treatment of BP may not be an appropriate choice of pre-treatment due to scanty growth of organism and little LCS conversion. The monosugars that were detected in SAE4-SB broth were arabinose, galactose, and cellobiose, and in SAE4-RH broth, glucose, xylose, mannose, fructose, and galactose were identified. In SAE4-BP, only galactose and cellobiose was identified.

A two-fold increase in ethanol concentration was observed in SAE4-SB and SAE4-RH with 1.21 and $1.0 \mathrm{~g} /$ $\mathrm{L}$, respectively, when compared to experimental setup 1 . Previous reports have claimed a maximum ethanol yield of 
Fig. 3 a Different growth pattern of Clostridium thermocellum in the standard cellulose and cellobiose media. In cellobiose media organism depicted an early catch up and increased $\mathrm{OD}_{600}$ values compared to cellulose media. However, the growth pattern was similar in both media. b Graphical representation of calculated $f$.RS from Clostridium thermocellum inoculated in cellobiose and cellulose medium. $f$.RS values from cellobiose media was $<1$, indicating its consumption by the organism and in cellulose media the $f$.RS value was $>1$ and increased each day indicating the release of reducing sugar due to hydrolysis exceeding the consumption rate of the organism

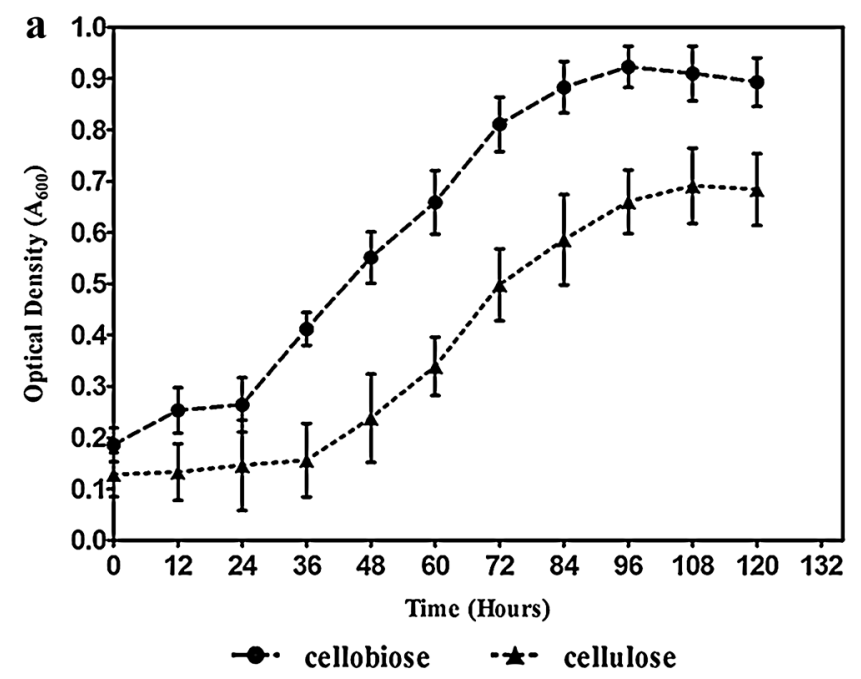

\section{b}

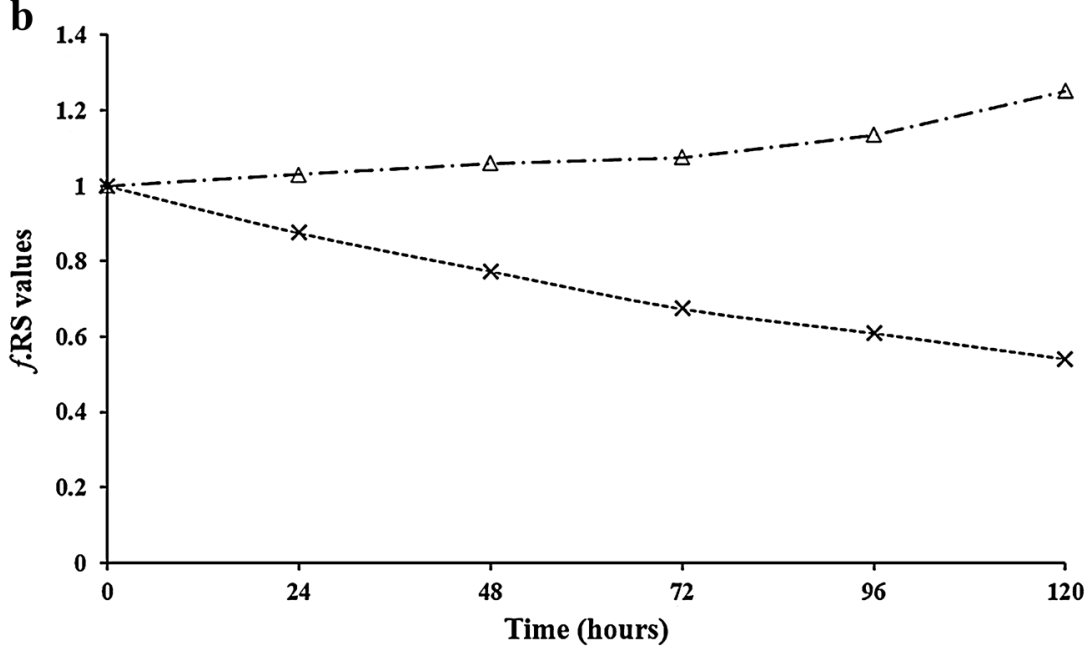

---x--- Cellobiose media $\quad-\backsim \cdot$ Cellulose media

Table 3 Comparative results of two experimental setups

\begin{tabular}{|c|c|c|c|c|c|}
\hline & Different media & Maximum O.D. ${ }^{a}$ & $f \cdot \mathrm{RS}^{\mathrm{b}}$ & Ethanol (g/L) & Residual recovery $(\%)$ \\
\hline \multirow[t]{3}{*}{ Experimental setup I } & SB & $0.415 \pm 0.017$ & 0.91 & 0.58 & $81.6 \pm 3.1$ \\
\hline & BP & $0.465 \pm 0.032$ & 0.65 & 0.3 & $82 \pm 2.4$ \\
\hline & RH & $0.518 \pm 0.034$ & 0.73 & 0.42 & $84.4 \pm 3.3$ \\
\hline \multirow[t]{3}{*}{ Experimental setup II } & SAE4-SB & $0.794 \pm 0.044$ & 1.18 & 1.21 & $36 \pm 2.3$ \\
\hline & SAE4-BP & $0.543 \pm 0.026$ & 1.01 & 0.42 & $74 \pm 3.0$ \\
\hline & PAE4-RH & $0.786 \pm 0.028$ & 1.38 & 1 & $48 \pm 2.2$ \\
\hline \multirow[t]{2}{*}{ Standard media } & DSM122 cellulose & $0.691 \pm 0.022$ & 1.25 & 1.14 & $35.7 \pm 1.1$ \\
\hline & DSM122 cellobiose & $0.956 \pm 0.030$ & 0.5 & 0.54 & - \\
\hline
\end{tabular}

Maximum OD as achieved for the batch

${ }^{\text {a }}$ Values are $\pm \operatorname{SE}(n=3)$

${ }^{\mathrm{b}} f$.RS $<1$ indicates that sugar consumption exceeded sugar release due to hydrolysis; $f$.RS $>1$ indicates that sugar release due to hydrolysis exceeded sugar consumption; and $f . \mathrm{RS}=1$ indicates that sugar released due to hydrolysis was equally consumed 
Table $4 f$.RS values of experimental setup II. $f$.RS were calculated at every $24 \mathrm{~h}$ interval for BP, SB, and RH

\begin{tabular}{|c|c|c|c|c|c|c|c|c|c|c|c|c|}
\hline \multirow[t]{2}{*}{ Hours } & \multicolumn{4}{|c|}{$f . \mathrm{RS}$ of $\mathrm{BP}$} & \multicolumn{4}{|c|}{$f . \mathrm{RS}$ of $\mathrm{SB}$} & \multicolumn{4}{|c|}{$f . \mathrm{RS}$ of $\mathrm{RH}$} \\
\hline & SAE1 & SAE2 & SAE3 & SAE4 & SAE1 & SAE2 & SAE3 & SAE4 & SAE1 & SAE2 & SAE3 & SAE4 \\
\hline 0 & 1.0 & 1.0 & 1.0 & 1.0 & 1.0 & 1.0 & 1.0 & 1.0 & 1.0 & 1.0 & 1.0 & 1.0 \\
\hline 24 & 0.94 & 0.95 & 0.90 & 1.03 & 0.98 & 0.99 & 0.99 & 0.95 & 0.96 & 0.91 & 0.88 & 1.03 \\
\hline 48 & 0.83 & 0.82 & 0.83 & 1.03 & 0.95 & 0.91 & 0.95 & 0.88 & 0.94 & 0.81 & 0.85 & 1.09 \\
\hline 72 & 0.71 & 0.78 & 0.71 & 1.08 & 0.82 & 0.88 & 0.89 & 0.97 & 0.81 & 0.83 & 0.81 & 1.30 \\
\hline 96 & 0.51 & 0.64 & 0.75 & 1.04 & 0.75 & 0.76 & 0.85 & 1.08 & 0.73 & 0.79 & 0.85 & 1.20 \\
\hline 120 & - & - & - & 1.01 & - & - & - & 1.18 & - & - & - & 1.38 \\
\hline
\end{tabular}

$1.4,0.83,0.77$, and $0.53 \mathrm{~g} / \mathrm{L}$ from various leafy substrates of Jamun (Syzygium cumini), bamboo, wild grass, and eucalyptus, respectively, using recombinant $C$. thermocellum cellulases (Mutreja et al. 2011). The present work claims an enhanced hydrolytic action of wild strain on SB and RH substrates with improved ethanol production without any genetic modification or externally added enzymes.

Consistent results were produced when inoculated into three consecutive batches having SAE4 composition. Here, the organism on acclimation became competent with a better hydrolytic capability and flourished in pre-treated LCS media without any growth enhancers. Simultaneous synergistic action of multi-enzyme complexes targeting a particular site of interest (Zverlov and Schwarz 2008) results in the formation of cellulose-enzyme-complexes (Mayer et al. 1987). Externally added enzyme-hydrolysis studies have been reported to get inhibited by its own enzyme products. Such inhibition is not exhibited in the case of synergistically acting multi-enzyme complexes ( $\mathrm{Lu}$ et al. 2006), which could likely be a contributing reason for increased yield of reducing sugar in this experimental setup.

\section{Conclusion}

Microbial hydrolysis is a mild and eco-friendly and costeffective approach for hydrolysis. The pure strain of Clostridium thermocellum was successfully acclimated by serially incubating in increasing concentrations of respective LCSs (gram equivalent cellulose) with cellobiose as growth inducer. The acclimated organism could then uninhibitedly grow in respective LCSs without any addition of the growth inducer and also showed ethanolgenesis comparable to the standard cellulose substrate. This study clearly establishes the need for acclimating Clostridium thermocellum to any LCS prior to fermentation. The acclimated organism, however, showed restraint in growing in BP medium likely due to the presence of undegraded lignin. The obtained results offer an alternative avenue for cost-effective and cleaner hydrolysis process prior to fermentation. Increasing the lignocellulosic load for the whole cell hydrolytic activity may increase the total reducing sugar pool which can lessen the dependency on techniques/ instruments needed to concentrate the same for ethanol fermentation. The microbial LCS hydrolysate thus obtained from agricultural waste residues can be a potential resource for biofuel. This slurry can also be fermented with other ethanolgenic organisms for enhanced ethanol titre without any added nutritional supplements.

Acknowledgements This work was supported by the Department of Science and Technology under Women Scientists Scheme A (SR/ WOS-A/LS-196/2011(G), and New Delhi for N.M. is gratefully acknowledged. We extend our gratitude to Shri AMM Muragappa Chettiar Research Centre, Chennai for UFLC analysis and SRM Nanotechnology Research Centre for FESEM analysis. We thank SRM University and management for all the facilities provided.

\section{Compliance with ethical standards}

Conflict of interest All authors declare that they have no conflict of interests.

Ethical statement This article does not contain any studies with human participants or animals performed by any of the authors.

Open Access This article is distributed under the terms of the Creative Commons Attribution 4.0 International License (http:// creativecommons.org/licenses/by/4.0/), which permits unrestricted use, distribution, and reproduction in any medium, provided you give appropriate credit to the original author(s) and the source, provide a link to the Creative Commons license, and indicate if changes were made.

\section{References}

Bilba K, Arsene M, Ouensanga A (2007) Study of banana and coconut fibers botanical composition, thermal degradation and textural observations. Bioresour Technol 98:58-68. doi:10.1016/ j.biortech.2005.11.030

Blum DL, Kataeva IA, Li X, Ljungdahl LG (2000) Feruloyl esterase activity of the Clostridium thermocellum cellulosome can be attributed to previously unknown domains of XynY and XynZ. J Bacteriol 182:1346-1351 
Brodeur G, Yau E, Badal K et al (2011) Chemical and physicochemical pretreatment of lignocellulosic biomass: a review. Enzyme Res 2011:1-17. doi:10.4061/2011/787532

Chaturvedi V, Verma P (2013) An overview of key pretreatment processes employed for bioconversion of lignocellulosic biomass into biofuels and value added products. 3 Biotech 3:415-431. doi:10.1007/s13205-013-0167-8

Das SP, Ghosh A, Gupta A et al (2013) Lignocellulosic fermentation of wild grass employing recombinant hydrolytic enzymes and fermentative microbes with effective bioethanol recovery. Biomed Res Int 2013:1-14. doi:10.1155/2013/386063

Desvaux L, Guedon E, Petitdemange $\mathrm{H}$ et al (2000) Cellulose catabolism by Clostridium cellulolyticum growing in batch culture on defined medium. Appl Environ Microbiol 66:2461-2470

Dharmagadda VSSS, Nokes SE, Strobel HJ, Flythe MD (2010) Investigation of the metabolic inhibition observed in solidsubstrate cultivation of Clostridium thermocellum on cellulose. Bioresour Technol 101:6039-6044. doi:10.1016/j.biortech.2010. 02.097

Dionisi D, Anderson JA, Aulenta F, Paton G (2014) The potential of microbial processes for lignocellulosic biomass conversion to ethanol: a review. J Chem Technol Biotechnol. doi:10.1002/jctb. 4544

Fleming RW, Quinn LY (1971) Chemically defined medium for growth of Clostridium thermocellum, a cellulolytic. Appl Microbiol 21:967

Guimarães JL, Frollini E, da Silva CG et al (2009) Characterization of banana, sugarcane bagasse and sponge gourd fibers of Brazil. Ind Crops Prod 30:407-415. doi:10.1016/j.indcrop.2009.07.013

Hörmeyer HF, Tailliez P, Millet J et al (1988) Ethanol production by Clostridium thermocellum grown on hydrothermally and organosolv-pretreated lignocellulosic materials. Appl Microbiol Biotechnol 29:528-535. doi:10.1007/BF00260980

Kim S, Dale BE (2004) Global potential bioethanol production from wasted crops and crop residues. Biomass Bioenergy 26:361-375. doi:10.1016/j.biombioe.2003.08.002

Kiyoshi K, Furukawa M, Seyama T et al (2015) Butanol production from alkali-pretreated rice straw by co-culture of Clostridium thermocellum and Clostridium saccharoperbutylacetonicum. Bioresour Technol 186:325-328. doi:10.1016/j.biortech.2015. 03.061

Kumar P, Barrett D, Delwiche MJ, Stroeve P (2009) Methods for pretreatment of lignocellulosic biomass for efficient hydrolysis and biofuel production. Ind Eng Chem Res 48:3713-3729

Lopez Hernandez J, González-Castro MJ, Alba IN, De La Cruz Garcia C (1998) High-performance liquid chromatographic determination of mono-and oligosaccharides in vegetables with evaporative light-scattering detection and refractive index detection. J Chromatograph Sci 36:293-298

Lu Y, Zhang Y-HP, Lynd LR (2006) Enzyme-microbe synergy during cellulose hydrolysis by Clostridium thermocellum. Proc Natl Acad Sci USA 103:16165-16169. doi:10.1073/pnas.0605381103

Luduena L, Fasce D, Alvarez VA, Stefani PM (2011) Nanocellulose from rice husk. BioResources 6:1440-1453

Lynd LR (1989) Production of ethanol from lignocellulosic materials using thermophilic bacteria: critical evaluation of potential and review. Adv Biochem Eng Biotechnol 38:1-52. doi:10.1007/ $\mathrm{BFb} 0007858$

Maki M, Leung KT, Qin W (2009) The prospects of cellulaseproducing bacteria for the bioconversion of lignocellulosic biomass. Int J Biol Sci 5:500-516

Mayer F, Coughlan MP, Mori Y, Ljungdahl LG (1987) Macromolecular organization of the cellulolytic enzyme complex of
Clostridium thermocellum as revealed by electron microscopy. Appl Environ Microbiol 53:2785-2792

Miller GL (1959) Use of dinitrosalicyclic acid reagent for determination of reducing sugar. Anal Chem 31:426-428

Mutreja R, Das D, Goyal D, Goyal A (2011) Bioconversion of agricultural waste to ethanol by SSF using recombinant cellulase from Clostridium thermocellum. Enzyme Res 2011:1-6. doi:10. 4061/2011/340279

Ndazi B, Karlsson S, Tesha J, Nyahumwa C (2007) Chemical and physical modifications of rice husks for use as composite panels. Compos Part A 38:925-935. doi:10.1016/j.compositesa.2006.07. 004

Nisha M, Shankar M, Krishnan N et al (2016) Direct estimation of ethanol as a negative peak from alcoholic beverages and fermentation broths by reversed phase-HPLC. Anal Methods 8:1-9. doi:10.1039/C6AY01075J

Pandey A (2011) Biofuels alternative feedstock and conversion process, 1st edn. Academic Press Inc., London

Paye JMD, Guseva A, Hammer SK et al (2016) Biological lignocellulose solubilization: comparative evaluation of biocatalysts and enhancement via cotreatment. Biotechnol Biofuels 9:8. doi:10.1186/s13068-015-0412-y

Pereira ALS, do Nascimento DM, Souza MSM et al (2014) Banana fibers composition. BioResources 9:7749-7763

Sindhu R, Binod P, Satyanagalakshmi K et al (2010) Formic acid as a potential pretreatment agent for the conversion of sugarcane bagasse to bioethanol. Appl Biochem Biotechnol 162:23132323. doi:10.1007/s12010-010-9004-2

Taherzadeh MJ, Karimi K (2008) Pretreatment of lignocellulosic wastes to improve ethanol and biogas production: a review. Int $\mathrm{J}$ Mol Sci 9:1621-1651. doi:10.3390/ijms9091621

Taylor MP, Eley KL, Martin S et al (2009) Thermophilic ethanologenesis: future prospects for second-generation bioethanol production. Trends Biotechnol 27:398-405. doi:10.1016/j. tibtech.2009.03.006

Tripathi SA, Olson DG, Argyros DA et al (2010) Development of pyrF-based genetic system for targeted gene deletion in Clostridium thermocellum and creation of a pta mutant. Appl Environ Microbiol 76:6591-6599. doi:10.1128/AEM.01484-10

Updegraff DM (1969) Semimicro determination of cellulose in biological materials. Anal Biochem 32:420-424. doi:10.1016/ S0003-2697(69)80009-6

Wang Z, Keshwani DR, Redding AP, Cheng JJ (2010) Sodium hydroxide pretreatment and enzymatic hydrolysis of coastal Bermuda grass. Bioresour Technol 101:3583-3585. doi:10.1016/ j.biortech.2009.12.097

Weimer PJ, Zeikus JG (1977) Fermentation of cellulose and cellobiose by Clostridium thermocellum in the absence of Methanobacterium thermoautotrophicum. Appl Environ Microbiol 33:289-297

Wilson DB (2011) Microbial diversity of cellulose hydrolysis. Curr Opin Microbiol 14:259-263. doi:10.1016/j.mib.2011.04.004

You C, Zhang X-Z, Sathitsuksanoh N et al (2012) Enhanced microbial utilization of recalcitrant cellulose by an ex vivo cellulosome-microbe complex. Appl Environ Microbiol 78:1437-1444. doi:10.1128/AEM.07138-11

Zverlov VV, Schwarz WH (2008) Bacterial cellulose hydrolysis in anaerobic environmental subsystems-Clostridium thermocellum and Clostridium stercorarium, thermophilic plant-fiber degraders. Ann N Y Acad Sci 1125:298-307. doi:10.1196/ annals. 1419.008 